\title{
Simultaneous Ring Voice-over-Internet Phone System Enables Rapid Physician Elicitation of Explicit Informed Consent in Prehospital Stroke Treatment Trials
}

\author{
Nerses Sanossian ${ }^{a}$ Sidney Starkman ${ }^{b}$ David S. Liebeskind ${ }^{b}$ Latisha K. Ali ${ }^{b}$ \\ Lucas Restrepo $^{b}$ Scott Hamilton ${ }^{c}$ Robin Conwit ${ }^{d}$ Jeffrey L. Saver ${ }^{b}$ \\ FAST-MAG Trial Investigators ${ }^{1}$ \\ ${ }^{a}$ Department of Neurology, University of Southern California, and ${ }^{b}$ Stroke Center and Department of Neurology, \\ University of California, Los Angeles, Calif., 'Stanford University, Palo Alto, Calif., and d Division of Extramural \\ Research, NIH/NINDS, Bethesda, Md., USA
}

\section{Key Words}

Prehospital trials $\cdot$ Stroke $\cdot$ Magnesium $\cdot$ Informed consent • Clinical trial

\begin{abstract}
Background: Cellular phone conversations between onscene patients or their legally authorized representatives (LARs) and off-scene enrolling physician-investigators require immediate and reliable connection systems to obtain explicit informed research consent in prehospital treatment trials. Methods: The NIH Field Administration of Stroke Therapy - Magnesium (FAST-MAG) Trial implemented a voiceover-internet protocol (VOIP) simultaneous ring system (multiple investigator cell phones called simultaneously and first responder connected to call) to enable physician-investigators to elicit consent immediately from competent patients or LARs encountered by 228 ambulances enrolling patients in a multicenter prehospital stroke trial. For 1 month, the number, origin, duration, and yield of enrolling line calls were monitored prospectively. Results: Six investigators were connected to 106 enrolling line calls, with no identified
\end{abstract}

unanswered calls. Thirty-five percent of new patient calls yielded an enrollment. The most common reasons for nonenrollment were last known well $>2 \mathrm{~h}(\mathrm{n}=7)$ and unconsentable patient without LAR available $(n=7)$. No non-enrollments were directly attributable to the VOIP system. In enrollments, consent was provided by the patient in $67 \%$ and a LAR in $33 \%$. The duration of enrollment calls (mean \pm SD: $8.4 \pm 2.5 \mathrm{~min}$, range 6-14) was longer than non-enrollment calls $(5.5 \pm 3.5$, range $2-13 ; p<0.001)$. The median interval from last known well to study agent start was 46 min, and $70 \%$ were enrolled within 60 min of onset. Conclusions: The simultaneous ring system was reliable and effective, permitting enrollment of a substantial number of patients within the first hour after stroke onset. VOIP cellular networks with simultaneous ring are a preferred means of facilitating consent in prehospital treatment trials.

Copyright $\odot 2009$ S. Karger AG, Basel

\section{KARGER}

๑ 2009 S. Karger AG, Basel

Fax +41613061234 E-Mail karger@karger.ch www.karger.com www.karger.com/ced
Nerses Sanossian, MD

Department of Neurology, University of Southern California

1200 N. State St., Room 5640

Los Angeles, CA 90033 (USA)

Tel. +1 323226 7383, Fax +1 323226 5869, E-Mail sanossia@yahoo.com 


\section{Introduction}

Physician elicitation of explicit informed consent by cell phone is an attractive method of enrolling competent prehospital patients and non-competent patients with on-scene legally authorized representatives (LARs) in trials of novel diagnostic and therapeutic interventions for hyperacute conditions, as it maximizes the autonomy of the research subjects $[1,2]$. However, a substantial technological challenge to prehospital cell phone consent elicitation is the requirement for an immediate phone connection between a remote physician-investigator and onscene personnel. If the physician-investigator is in a hospital area or geographic region which does not have cell reception, the emergent enrollment call may go unanswered and the opportunity for entry into the study be missed.

Recently commercialized voice-over-internet protocol (VOIP) simultaneous ring phone systems are a potential solution to this challenge, permitting routing of field calls immediately to several physician-investigator cell phones simultaneously, with the first responding physician immediately connected to the field. 'Simultaneous ring' refers to a function in the user settings of the VOIP system website, which allows multiple physician-investigator telephone numbers to be entered into a list on the VOIP provider website. When the VOIP telephone number is called, rather than a single phone connection, all telephone numbers listed are rung and only the first phone to respond is connected (fig. 1).

This study evaluated the effectiveness of a VOIP simultaneous ring system in enabling enrollments in a multicenter National Institutes of Health prehospital clinical trial.

\section{Methods}

The Field Administration of Stroke Therapy - Magnesium (FAST-MAG) trial is a phase-3 NIH-NINDS-sponsored randomized placebo-controlled clinical trial of field-initiated magnesium sulfate in hyperacute stroke patients within the first $2 \mathrm{~h}$ of defined symptom onset. Participating sites are 31 emergency medical system agencies, 228 ambulances, and 46 acute receiving hospitals in the County of Los Angeles. Explicit informed consent is obtained via cellular phone conversation between on-scene patients or LARs and off-scene enrolling physician-investigators. In the state of California, the following individuals can act as a LAR for research: spouse, father, mother, sibling, son, daughter, domestic partner, advanced health care directive agent, or health care conservator.
When paramedics identify a patient who meets the study inclusion criteria, they tell the patient/LAR that the patient is a potential candidate for a research clinical trial of a new treatment for stroke and ask if the patient/LAR are interested in speaking to a physician-investigator to learn more about the study. If the answer is affirmative, the paramedics hand the written consent form to the patient/LAR, use the in-vehicle cell phone or an onscene landline to reach a FAST-MAG physician-investigator, and provide the physician with a brief report on patient status. Criteria for the physician-investigator to proceed with consent elicitation are that the patient meets all study entry criteria besides the provision of informed consent. Each ambulance carries 8 study consent forms, 1 each in English and Spanish for the 4 destination hospitals to which it most commonly transports patients. The invehicle cell phone is preprogrammed with the phone numbers for the trial English-speaking physician enrolling line and Spanishspeaking physician enrolling line.

On a rotating basis, 1 investigator is assigned to be first call responder, who will respond immediately to any call on the dedicated FAST-MAG cell phone. The other investigators are designated back-ups, who will pick up the call if the first responder has not answered within 4 rings. At all times, up to 5 Anglophone physician investigators are on call to respond to English line enrollments and 3 Hispanophone physician investigators are on call to respond to Spanish line enrollments. All enrollments are carried out in parallel with routine paramedic evaluation, preparation, and transport.

In the FAST-MAG pilot trial, standard point-to-point cell connections were employed to connect consent providers in the field with off-scene enrolling physicians [1,3]. If the first physician's cell phone did not respond, the call was forwarded to the second on-call physician, and then the third. This system worked fairly effectively; however, occasionally strings of delayed call forwarding resulted in paramedic call abandonment and a missed opportunity to enroll a patient. For the NIH FAST-MAG main phase 3 trial, we therefore deployed a simultaneous ring VOIP call routing system. The trial used a standard off-the-shelf VOIP commercial service, including the services provided by Vonage and ATT. English and Spanish enrolling line calls are directed to a VOIP virtual number and then simultaneously forwarded to the dedicated trial cell phones of up to 5 on call physician-investigators fluent in the language of the consent provider. Physician-investigators are not required to initiate direct contact with paramedics, and only the first physician responder is connected.

For this intensive study of system performance, all phone calls through the Vonage VOIP system to the enrolling Spanish and English lines were recorded into a database for the month of July 2007. The origin, duration, purpose, number, and results of calls were noted. A hot and cold pursuit method was used to identify any missed calls. The VOIP system automatically generated call logs that were reviewed daily. Results of each call, including enrollment and non-enrollment outcomes, were collected from physician-investigators. Among enrolled patients, demographic and symptom information was documented by on-call research nurse coordinators who responded to the receiving hospital immediately upon completion of field enrollment. All 6 enrolling physician-investigators are board-certified/eligible neurologists with subspecialty expertise in vascular neurology, and 3 are fluent in Spanish. 


\section{Chain Cell Forwarding System}
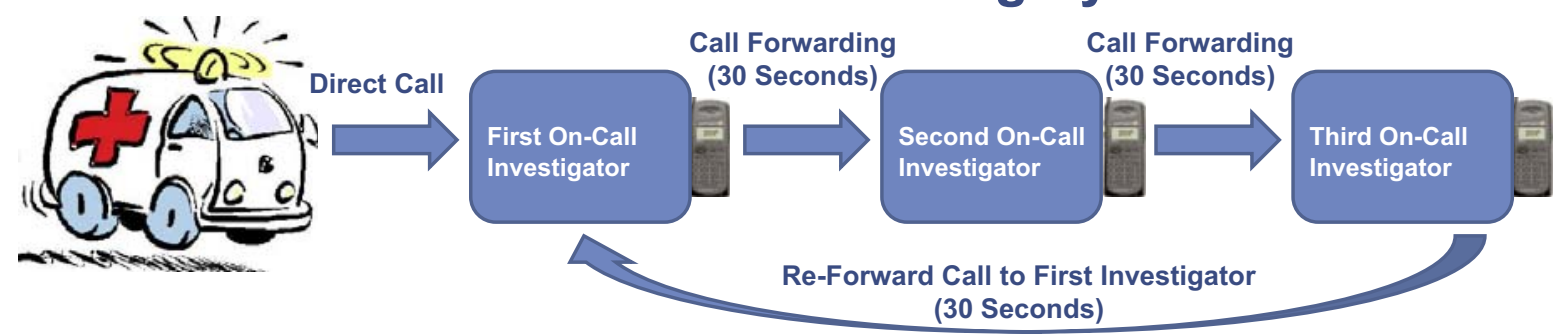

(30 Seconds)

\section{- تr'á:-Voice-Over-Internet Phone (VOIP) System}
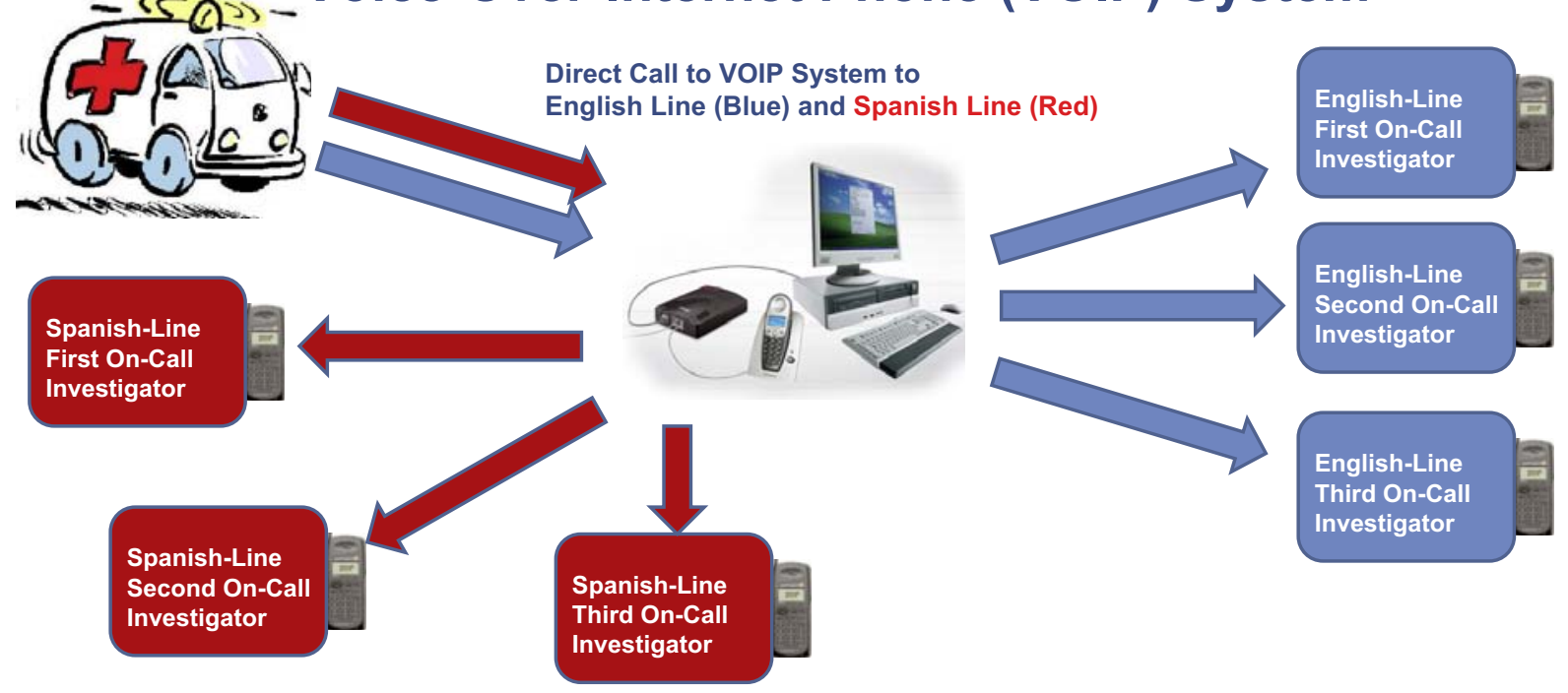

Fig. 1. Diagram comparing the previously-used daisy-chain call forwarding system with the current FASTMAG voice-over-internet simultaneous ring phone system.

\section{Results}

During the month of intensive VOIP system evaluation, the 6 physician-investigators were successfully connected to 106 enrolling line calls (table 1). Hot and cold pursuit surveillance identified no calls that went unanswered. Among this cohort, the median time from last known well to start of study drug was $46 \mathrm{~min}$ and $70 \%$ of patients were enrolled within the first hour after onset. Overall, during the first 4 years of trial performance, 760 patients have been enrolled in the NIH FAST-MAG Trial using the VOIP simultaneous ring system.

Of the 106 calls, 52 (49\%) were initial calls from paramedics regarding a potential enrollment. Other types of calls were: (1) paramedic callbacks from dropped calls (3\%), (2) paramedic callbacks with additional questions regarding enrolled patients (3\%), (3) non-urgent trial-related calls with non-enrollment study questions (33\%), and (4) wrong numbers or calls unrelated to the trial (11\%; table 1). Paramedic calls from the field originated from emergency-medical-services-supplied ambulancebased cell phones in all but 1 case, in which a home landline was employed. During the intensive study month, 1 call for a potential enrollment was made not to the dedicated VOIP enrolling lines, but instead to a separate study hotline phone number established for general non-enrollment questions. 
Table 1. Characteristics and outcomes of all FAST-MAG calls over 1 month of intense monitoring

\begin{tabular}{lc}
\hline Characteristic & $\mathrm{n}$ \\
\hline Total number of calls & 106 \\
\hline $\begin{array}{l}\text { Origin of call } \\
\text { EMS initial call for potential enrollment }\end{array}$ & $52(50)$ \\
EMS callbacks after dropped initial calls & $3(3)$ \\
EMS callbacks with additional questions following & $3(3)$ \\
$\quad$ enrollment & $35(33)$ \\
Non-urgent non-enrollment trial questions & $12(11)$ \\
\hline $\begin{array}{l}\text { Results of initial enrollment calls }(n=52) \\
\text { Study enrollment (of all initial calls) }\end{array}$ & $18(35)$ \\
Non-enrollment & $34(65)$ \\
\hline $\begin{array}{l}\text { Reasons for non-enrollment } \\
\text { Last known well time not established } \leq 2 \mathrm{~h}\end{array}$ & 7 \\
$\begin{array}{l}\text { No LAR available } \\
\text { Systolic blood pressure }>220 \text { mm Hg }\end{array}$ & 7 \\
Subject competent and declined \\
Preexisting condition which would interfere with & 4 \\
$\quad$ outcome assessment & 3 \\
\hline
\end{tabular}

Figures in parentheses are percentages. EMS = Emergency medical services.

Eighteen of the 52 enrolling line initial calls regarding potential study subjects yielded an enrollment (35\%). Enrollment process occurred in parallel with, rather than serial to, standard prehospital care in order to reduce the possibility of delay in on-scene to emergency department door time. The mean duration of enrollment calls was 8.4 $\pm 2.5 \mathrm{~min}$ (range 6-14), which was longer than non-enrollment calls (5.5 $\pm 3.5 \mathrm{~min}$, range $2-13, \mathrm{p}<0.001)$. The most common reasons for non-enrollment were inability to clearly identify a last known well time of $\leq 2 \mathrm{~h}$, the patient being deemed not competent to consent and no LAR available, and systolic blood pressure $>220 \mathrm{~mm} \mathrm{Hg}$ (table 1).

Trial enrollment activities by the enrolling physician were performed simultaneously with standard paramedic activities in the field. As a result of this parallel, rather than serial, approach, enrollment activities in the field did not cause prolonged delay in patient delivery to receiving hospitals. The median interval from paramedic arrival on the scene to patient emergency department arrival was $34 \mathrm{~min}$, which is at the low end of the national average for time in the field for stroke patients.
Subject location at the time of the enrollment conversation from the field was most often the subject's own home (61\%). However, enrollments also occurred outdoors after bicycle riding, at a lecture hall, in a church bingo hall, in a casino, and on the shoulder of the interstate freeway.

No non-enrollments were directly attributable to the VOIP system. There were 3 dropped calls due to poor cellular phone reception; in all cases, contact was immediately re-established by paramedic callback. Two non-enrollments were due to competent patients being hard of hearing, and therefore being unable to participate in the informed consent process via cell phone.

Of the 18 enrollments in July 2007, 12 patients provided self consent and 6 were enrolled by a LAR because the subject was not competent to provide consent due to aphasia, anosognosia, or other cognitive deficits. The mean age of enrolled patients was 76 years, and $44 \%$ were female. There were no cases in which patients or LAR withdrew consent upon arrival to the hospital.

\section{Discussion}

More than 50 neuroprotective agents have been tested in clinical trials of acute stroke, but none has demonstrated efficacy in a definitive phase 3 trial $[4,5]$. A repeatedly identified design defect of past trials is failure to initiate neuroprotective therapy early after symptom onset, when the volume of potentially salvageable brain tissue is maximal. Paramedic initiation of experimental therapy in the field is a promising new strategy in focal stroke treatment trials, enabling the earliest possible delivery of trial therapy. One potential limitation of this method is the technical feasibility of obtaining informed consent from competent patients in the field. We found that a simultaneous ring VOIP system was reliable and effective, permitting enrollment of a substantial numbers of patient within the first hour after stroke onset.

The most commonly used method of enrolling patients in field treatment trials is via waiver of explicit consent mechanism, after performing a process of public disclosure and community consultation [6, 7]. This enrollment process was designed for use in patients who have been rendered non-competent by their emergency condition, such as cardiac arrest, status epilepticus, severe head trauma, or severe focal stroke [7]. Whether this enrollment mechanism can be employed in patients who retain competency and the ability to decide for themselves whether or not they would like to participate in a research trial has not yet been addressed by the federal legal sys- 
tem. Ethically, obtaining the explicit consent of the research subject or their LAR is always preferable to employing waiver of explicit consent, as it maximizes patient autonomy and respect for persons [8].

There are several mechanisms of obtaining elicit explicit consent in the field, each with advantages and disadvantages: (1) Paramedics can elicit research informed consent. This approach is the simplest to implement and was employed successfully in trials performed through the early years of this decade, including studies of prehospital thrombolysis for myocardial infarction $[9,10]$. However, there are potential practical, regulatory, and ethical issues. Tying paramedics up in consent elicitation may distract them from performing their routine prehospital care activities rapidly and effectively. Federal regulations now require that research consent elicitors complete formal training in ethics of research and research consent, yet having all paramedics in a system complete and maintain Federal certification in research ethics is a considerable challenge. Also, paramedics are not experts in the experimental therapy being tested or the condition being treated, and may not be able to answer authoritatively questions that patients or LARs have about the trial. (2) A short-form initial consent can be employed [11]. Under this mechanism, patients or LARs would read and sign a brief initial consent document in the field that summarized only the most critical aspects of study participation. Study procedures would then be initiated. Upon hospital arrival, patients or LARs would then meet with a physician-investigator and review and sign a standard full-length consent form, confirming consent for full study participation. Drawbacks of this approach include incomplete patient/LAR understanding of the study at the time of study initiation and the potential for a substantial rate of subject dropout upon learning full study details after hospital arrival, introducing noise and the potential for bias. (3) Physician-investigator cell phone elicitation of consent in the field [1]. This approach allows the patient/LAR to have their study-related questions definitively answered by a study investigator prior to enrollment and permits paramedics to continue to perform their prehospital care duties unimpeded while the enrollment process is taking place. Cell phone consent elicitation thereby maximizes patient autonomy and minimizes the impact of study performance upon routine prehospital care. The only modest drawback of this approach in its initial implementation was occasional delays in call response and call abandonment due to point to point cell phone connections and a serial call-forwarding system.

FAST-MAG Voice-over-Internet Phone System
Standard commercial VOIP simultaneous ring systems resolve this minor difficulty with field cell phone consent elicitation, directing ambulance calls in parallel to up to 5 on call physician-investigators simultaneously and connecting the first responding investigator immediately to the field. Using this system, all acute enrolling calls from the field were successfully answered and handled by an enrolling physician-investigator during the study period. One third of first-enrolling calls yielded a study enrollment. The ability to obtain consent from an on-scene LAR was an important aid to study enrollment, increasing the proportion of enrollments by $50 \%$ during the study month, in agreement with prior studies $[12,13]$. The field enrollment process did permit enrollment of a substantial proportion of patients in the first $1-2 \mathrm{~h}$ after symptom onset, a time window not previously achieved in acute stroke trials. Recent neuroprotective trials using standard post-hospital arrival enrollment enrolled only $0.2 \%$ of patients in the first hour after symptom onset [14]; in the FAST-MAG trial, more than $70 \%$ of patients were enrolled within the first hour.

For clinical trials which rely on immediate contact between paramedics in the field and physicians, a multiple pager system is suboptimal because of the inherent delays between a page being made and the call-back. Having paramedics directly call a single on-call investigator's cell phone is impractical, because that investigator may be unreachable, due to rounding in a hospital ward where cell phones are prohibited, traveling through an area with incomplete cell network coverage, or engaged in activities precluding immediate response (bathing, etc). The described VOIP simultaneous ring system is unique because it allows for immediate contact between paramedics and investigators without any delay. In addition, the response reliability will depend on the number of physicians whose cell phones ring simultaneously. For example, if 5 phones ring simultaneously, it is more likely that at least 1 of the investigators will immediately be in a position where they can answer the call, take notes, have a conversation with the patient or LAR and then go through the series of actions required after an enrollment.

The VOIP system is a solution to problems related to responding physician-investigators passing through an area of poor cell phone coverage and spending time on hospital wards which prohibit cell phone use. The system does not resolve issues with paramedic initiators being in areas with poor cell phone reception, which has been a rarely encountered problem. If time allows, paramedics may use on-scene land lines to contact enrolling physician-investigators in this situation.

Cerebrovasc Dis 2009:28:539-544 
This study has limitations. Although all phone calls to the trial VOIP simultaneous ring system were documented to be successfully connected, we do not have data on the duration between paramedic call placement and physician answer, although we would suspect that it was seconds. Also only qualitative, not quantitative, direct comparisons could be made between the current VOIP simultaneous ring system and the previous point to point call-forwarding system because formal data on the point to point call system was not prospectively collected.
The simultaneous ring VOIP system was reliable and effective, permitting enrollment of a substantial number of patients within the first hour after stroke onset. Simultaneous ring VOIP should be a preferred means of facilitating consent in prehospital treatment trials for conditions in which patients retain research decision-making competency.

\section{Acknowledgments}

This work was supported by NIH-NINDS Award U01 NS 44364

\section{References}

-1 Saver JL, Kidwell C, Eckstein M, Ovbiagele B, Starkman S: Physician-investigator phone elicitation of consent in the field: a novel method to obtain explicit informed consent for prehospital clinical research. Prehosp Emerg Care 2006;10:182-185.

$\checkmark 2$ Barsan WG, Pancioli AM, Conwit RA: Executive summary of the National Institute of Neurological Disorders and Stroke conference on Emergency Neurologic Clinical Trials Network. Ann Emerg Med 2004;44:407412.

-3 Saver JL, Kidwell C, Eckstein M, Starkman S: Prehospital neuroprotective therapy for acute stroke: results of the Field Administration of Stroke Therapy-Magnesium (FASTMAG) pilot trial. Stroke 2004;35:e106-e108.

-4 Ovbiagele B, Kidwell CS, Starkman S, Saver JL: Potential role of neuroprotective agents in the treatment of patients with acute ischemic stroke. Curr Treat Options Cardiovasc Med 2003;5:441-449.

5 Savitz SI, Fisher M: Future of neuroprotection for acute stroke: in the aftermath of the SAINT trials. Ann Neurol 2007;61:396402.
6 Biros MH: Research without consent: exception from and waiver of informed consent in resuscitation research. Sci Eng Ethics 2007; 13:361-369.

7 Saver J, American Stroke Association: Food and Drug Administration public hearing on the conduct of emergency clinical research testimony of the American Stroke Association. Acad Emerg Med 2007; 14:e57-e58.

8 National Commission for the Protection of Human Subjects of Biomedical and Behavioral Research: The Belmont Report: Ethical Principles and Guidelines for the Protection of Human Subjects Research. Washington, Department of Health, Education and Welfare, 1978.

-9 Steg PG, Bonnefoy E, Chabaud S, Lapostolle F, Dubien P-Y, Cristofini P, Leizorovicz A Touboul P, Comparison of Angioplasty and Prehospital Thrombolysis in Acute Myocardial Infarction (CAPTIM) Investigators: Impact of time to treatment on mortality after prehospital fibrinolysis or primary angioplasty: data from the CAPTIM randomized clinical trial. Circulation 2003;108:28512856.
10 Rosenberg DG, Levin E, Lausell A, Brown A, Gardner J, Perez E, Veenendaal M, Ong YS, Gunn M: Feasibility and timing of prehospital administration of reteplase in patients with acute myocardial infarction. J Thromb Thrombolysis 2002;13:147-153.

11 Iwanowski P, Budaj A, Czlonkowska A, Wasek W, Kozlowska-Boszko B, Oledzka U, Maselbas W: Informed consent for clinical trials in acute coronary syndromes and stroke following the European Clinical Trials Directive: investigators' experiences and attitudes. Trials 2008;9:45.

12 Saver JL, Starkman S, Fox S, Saver R, Biller J: The impact upon clinical stroke trials of restricting informed consent. Stroke 1995;26: 157.

13 Flaherty ML, Karlawish J, Khoury JC, Kleindorfer D, Woo D, Broderick JP: How important is surrogate consent for stroke research? Neurology 2008;71:1562-1563.

14 Ferguson KN, Kidwell CS, Starkman S, Saver JL: Hyperacute treatment initiation in neuroprotective agent stroke trials. J Stroke Cerebrovasc Dis 2004;13:109-112. 